\section{Hallucinations and nonsensory correlates of neural activity}

\author{
Kenneth D. Harris \\ Center for Molecular and Behavioral Neuroscience, Rutgers, The State \\ University of New Jersey, Newark, NJ 07102. \\ kdharris@andromeda.rutgers.edu http://qneuro.rutgers.edu
}

Abstract: Behrendt \& Young (B\&Y) suggest that hallucinations occur as a result of decoupling of neuronal populations from sensory control. I propose that such a decoupling is in fact a constant feature of brain activity, even under nonpathological conditions. This position is justified by evidence from recent neurophysiological recording studies. I suggest that hallucinations arise because of a breakdown in segregation of internally and externally generated activity in a neuronal population.

The core of Behrendt \& Young's (B\&Y's) thesis is that, during hallucinations, neuronal activity in sensory cortex "decouples" from afferent input - rather than reflecting the structure of the outside world, sensory neurons take on a life of their own, forming activity patterns only partially influenced by ongoing sensory stimulation. This is an intriguing explanation for the phenomenon of hallucinations. The aim of this commentary is to suggest that such a decoupling may in fact be a constant feature of brain function, including during nonpathological conditions. The following discussion will describe recent neurophysiological experiments that support this position.

The idea of a decoupling of neural activity from strict sensory control goes at least as far back as the "cell assembly" hypothesis of Hebb (1949). The cell assembly is defined as a subset of neurons among which excitatory connections have been strengthened by repeated coactivation, allowing the assembly to later maintain its activity through reverberant mutual excitation. A cell assembly may be activated by sensory stimulation, or it may also fire as a result of purely internal factors, such as the prior activity of another assembly. The resulting "phase sequence" of active assemblies was Hebb's model for volitional cognitive activity (the "train of thought"). In contrast to contemporaneous "feed-forward" theories of brain function, which concentrate on the production of simple stimulus-response associations, Hebb's theory also allows for behavior resulting purely from internal activity, whereby the phase sequence of active assemblies, decoupled from sensory control, lead eventually to the production of motion.

Although this theory has had a profound effect on thinking about the brain over the last half century, it has only recently become possible to test it directly at the spike-train level. The spike trains of neurons in sensory systems are certainly correlated with the structure of sensory input. However, repeated presentations of identical stimuli lead to varying responses across repetitions (Britten et al. 1993; Mazurek \& Shadlen 2002; Shadlen \& Newsome 1998; Softky \& Koch 1993; Stevens \& Zador 1998). This variability may simply be noise (Shadlen \& Newsome 1994; 1998; Zohary et al. 1994), or it may reflect an essential element of brain function. Indeed, any division into "signal" and "noise" itself rests on assumptions. The "signal" is typically defined to be the stimulus presented by the experimenter, and the "noise" to be any further variability, assumed random and not helpful for information processing. Alternatively, the variability may also represent a "signal," just not one under experimental control. In particular, variability may reflect participation of neuronal assemblies in cognitive processes not directly related to the stimulus presented by the experimenter.
How might this possibility be tested experimentally? If a cortical region is involved in suprasensory processing, it should happen not only in a single cell, but also at the level of the whole population. To distinguish the involvement of neuronal assemblies in processing nonsensory information from stochastic noise, therefore, requires the recording and analysis of large cell populations, where suprasensory processing will be reflected by coordination of spike trains beyond that predicted by common stimulus modulation.

Recordings from neuronal pairs have revealed that coincident spikes occur frequently in a wide variety of systems. Two types of pairwise synchronization are commonly seen, with characteristic time scales of $\sim 1$ msec (Constantinidis et al. 2001; Csicsvari et al. 1998; Usrey \& Reid 1999) and 25 msec (Bair et al. 2001; Constantinidis et al. 2001; DeCharms \& Merzenich 1996). It has been hypothesized that 1-msec scale synchronization reflects monosynaptic drive between neurons (Csicsvari et al. 1998; Marshall et al. 2002) or common monosynaptic input from a third cell (Usrey \& Reid 1999), whereas 25-msec scale synchrony results from more general network coordination (Constantinidis et al. 2001).

What role might this 25 -msec synchrony play in neural processing? In a recent study (Harris et al. 2003), we investigated the hypothesis that this coordination reflects an organization into assemblies whose activity can reflect both external sensory input and internal cognitive processes. One signature of the assembly organization is the existence of anatomically distributed groups of neurons, whose activity is synchronized more than predicted by common sensory modulation. A second postulated signature is that, although individual neurons may participate in many assemblies, not every possible combination of cells comprises a cell assembly. The latter feature should be reflected by a statistical preference in the probability with which a neuron joins its various peers in synchronous firing. A novel "peer prediction method" was used to show that precisely such an organization exists in the hippocampus of rats exploring a spatial environment. Neurons are organized into assemblies, whose timing and composition could not be predicted simply from the animal's trajectory through space (Harris et al. 2003). Furthermore, the time scale of this assembly organization could also be estimated, and was found to be $10-30 \mathrm{msec}$. This time scale may be of particular physiological significance. Because it closely matches the membrane time constant of pyramidal neurons in the hippocampal region, activity synchronized with this time scale may be optimal for inducing spiking in downstream neurons. Furthermore, this time scale matches the period of the hippocampal gamma oscillation and the effective window for synaptic plasticity. We therefore suggest that assembly activity may be optimal for propagation and storage of information in neuronal circuits.

These results suggest an extension of B\&Y's hypothesis. That is, even under nonpathological conditions, neuronal assemblies are equally involved in internal cognitive processes, such as mental imagery, as in the representation of the external world. The question is then: How does the healthy brain keep the internally generated and external worlds separate? When a neuron fires a spike, how do postsynaptic cells know whether that spike coded for an external sensory event or an internally generated one? This task may be harder than we imagine, and the breakdown of this balancing act may be the cause of hallucinations. 